\title{
UNCECOMP 2015
}

\section{$1^{\text {st }}$ International Conference on Uncertainty Quantification in Computational Sciences and Engineering}

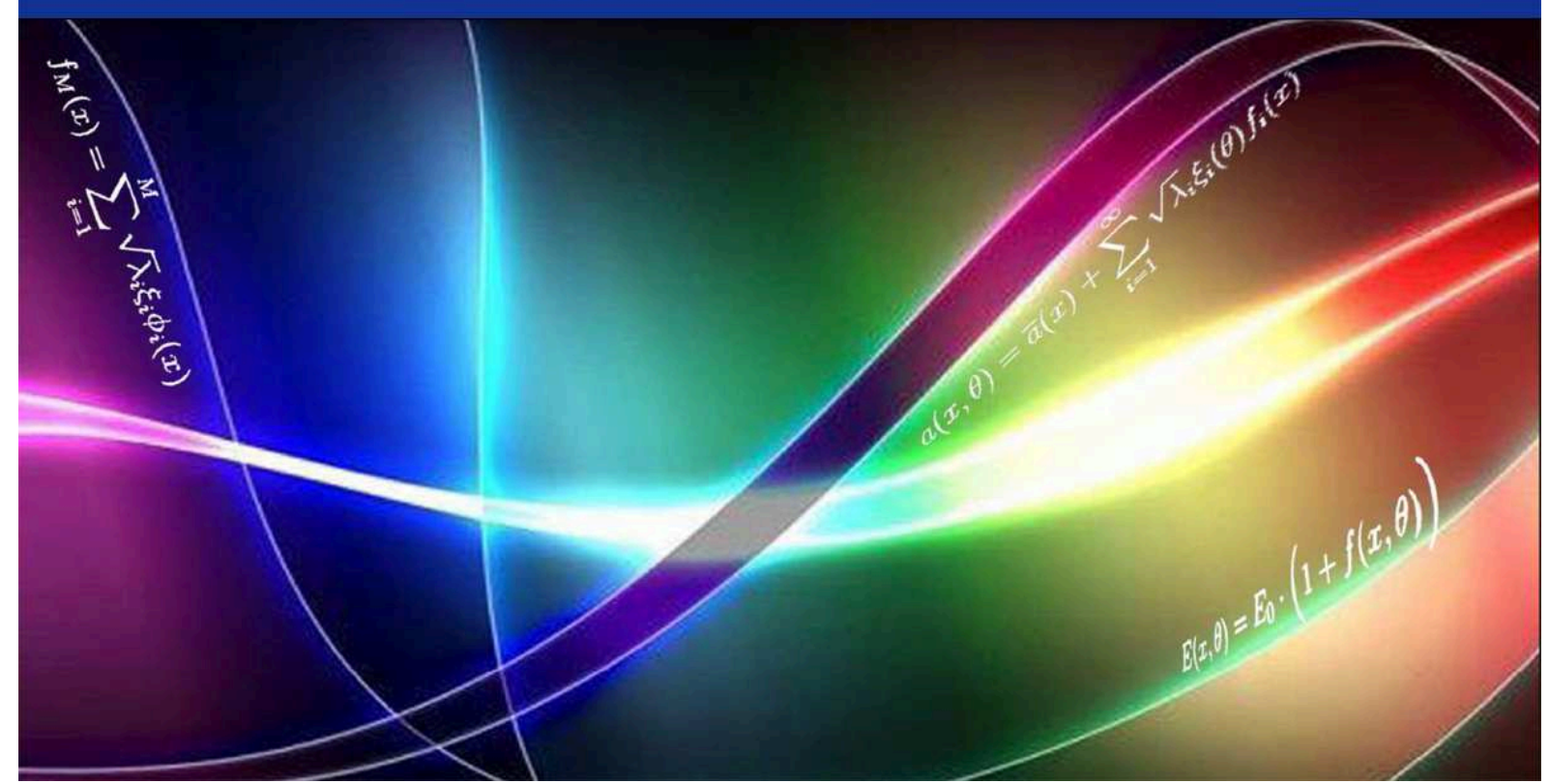

Ferrari, R., Pioldi, F., Rizzi, E., Gentile, C., Chatzi, E., Klis, R., Serantoni, E., Wieser, A., (2015),

"Heterogeneous sensor fusion for reducing uncertainty in Structural Health Monitoring", Proceedings of the 1st ECCOMAS International Thematic Conference on Uncertainty Quantification in Computational Sciences and Engineering (UNCECOMP 2015), Editors: M. Papadrakakis, V. Papadopoulos, G. Stefanou, Crete Island, Greece, 25-27 May 2015;

Proceedings, ISBN: 978-960-99994-9-6, p. 511-528 (18 pages). 


\title{
HETEROGENEOUS SENSOR FUSION FOR REDUCING UNCERTAINTY IN STRUCTURAL HEALTH MONITORING
}

\author{
R. Ferrari ${ }^{1{ }^{*},}$ F. Pioldi ${ }^{1}$, E. Rizzi ${ }^{1}$, C. Gentile ${ }^{2}$, E. Chatzi ${ }^{3}$, R. Klis ${ }^{3}$, \\ E. Serantoni ${ }^{3}$, A. Wieser ${ }^{3}$ \\ ${ }^{1}$ Università di Bergamo, I-24044 Dalmine (BG), Italy \\ e-mail: rosalba.ferrari@unibg.it \\ ${ }^{2}$ Politecnico di Milano, I-20133 Milano, Italy \\ ${ }^{3}$ ETH Zürich, CH-8093 Zürich, Switzerland
}

Keywords: Structural Health Monitoring (SHM), Laser scanning, QDaedalus, Wireless sensors, Displacement measurements, Data Fusion, Structural identification.

\begin{abstract}
This work attempts a comprehensive processing of response signals acquired from an experimental campaign on a local historical bridge using four different types of sensory systems. A general analysis setup is developed, allowing for consistent modal dynamic identification via individual signal processing as well as via data fusion through dedicated MultiRate Kalman filtering approach. The investigation reveals the potential and limitations in terms of utilization of novel instrumentation systems to be adopted for Structural Health Monitoring (SHM) purposes.
\end{abstract}




\section{INTRODUCTION}

Monitoring dynamic behavior, evaluating serviceability, detecting diffused and localized damage and estimating residual performance capacity of civil infrastructures appear nowadays as crucial aspects of modern structural assessment within typical Structural Health Monitoring (SHM) purposes. The need for realization of aforementioned goals pushes towards the development of increasingly reliable and efficient instrumentation devices, for consistent detection and measurement.

During the last decades, innovative sensor systems such as wireless sensors and noncontact instruments have surfaced as appropriate means for monitoring large infrastructural systems, such as bridges. The use of these technologies still lies under exploration. Hence, they are often employed together with more traditional recording approaches, such as by standard accelerometers. This allows to discover the true potential of novel instrumentation devices and to enrich or complement the information acquired from standard measurement systems.

This paper deals with the analysis and reliability of in-situ experimental data, collected during an experimental campaign on a historical (1917) Reinforced Concrete (RC) arch bridge located on the Adda river between Brivio (province of Lecco) and Cisano Bergamasco (province of Bergamo), Lombardia region, Italy [13]. A three-day measurement campaign on the bridge took place on June 2014. In that, four different types of instrumentation for dynamic testing have been employed. In particular, a laser scanner, a wireless MEMS sensor system, total stations (QDaedalus instrumentation) [4] and a system of conventional high-sensitivity accelerometers have been simultaneously adopted, in order to extract information concerning the dynamic response of the structure under Ambient Vibration Testing (AVT) conditions. AVT proves as a suitable approach to the identification of the dynamic characteristics of the structure, since, due to traffic conditions, the bridge is sufficiently excited under operational loads.

On the reporting of this work is organized in two companion papers. Work [6] presents the detailed AVT performed with conventional high-sensitivity accelerometers and the development of updated Finite Element models of the bridge with different levels of complexity and refinement, specifically in the prediction of modal properties, while the current work focuses on the analysis of the various data coming from the different adopted instrumentation systems, accounting also for data fusion and for reliability assessment of the acquired data.

Among the sensor systems that have been deployed on the bridge, wired accelerometers surely represent the more reliable and well-known instrumentation solution. In fact, although Wireless Sensor Networks (WSN) and displacement sensors are not novel in the SHM field, their use is not yet as widely established as that of wired accelerometers. However, the discovery of new "smart" systems that may alert and trigger up-to-date remedial actions on existing infrastructures when needed, or that may reduce maintenance and inspection costs, comprises currently an intensely pursued research goal. Within such a context, WSN systems and optical methods for displacement measurements, namely the QDaedalus system and Laser Scanning data, are cross-assessed herein along the well-established tethered accelerometer solution.

Referring to non-contact displacement measurement systems, the former system, i.e., the QDaedalus is a measurement system developed at the Institute of Geodesy and Photogrammetry at ETH Zurich. The basic idea is to replace the eye-piece of an existing total station by a CCD camera in a non-destructive way in order to measure fully automatically very accurate spatial directions to visible objects without using corner-cube targets as in standard Automatic Object Recognition (AOR). A Z+F IMAGER ${ }^{\circledR} 5006$ i Laser Scanner was used as a second op- 
tion for obtaining dynamic displacement measurements. The laser scanner solution is herein explored as an option which bears significant potential for future use, since it provides the unique potential of both defining the geometry of the monitored structure, via geometrical scan, while at the same time carrying out structural identification tasks. In this way, the FE modeling of a structure and its subsequent FE model updating could be performed "on-thefly" within a single integrated device.

The employment of "new" measurement systems within a comprehensive experimental campaign such as the one presented herein represents an opportunity to investigate and compare the performance of these systems. In this paper, the campaign outcomes are collected and investigated in terms of their reliability towards detecting the current modal characteristics of the bridge. The wired accelerometers are considered as the main reference acquisition system and the modal properties identified from their signals are assumed as the identification target for the further instrumentation systems.

Moreover, possibilities of data fusion are explored by combining data coming from the wired accelerometers and from the QDaedalus dynamic displacement measurement system, namely by recombining acceleration and displacement response signals, sampled at different rates. Towards this end, a Multi-Rate Kalman filtering [5,14] technique has been developed, with the main purpose of investigating the potential of displacement measurements, in terms of independent or complementary modal dynamic identification of the dynamic characteristics of the bridge.

The paper is structured as follows. Section 2 briefly presents the infrastructure of interest, namely the Brivio bridge (1917). Section 3 illustrates the experimental campaign performed on the bridge, by describing the measurement layout adopted for each employed instrumentation. In Section 4, the outcomes obtained from the response of the structure under AVT are presented, in terms of modal properties of the bridge, identified in the frequency domain through the use of different Frequency Domain algorithms $[1,3,10]$. In particular, the identified modal frequencies are presented, as identified via the data acquired from each instrumentation solution deployed on the bridge, along with some statistical data related to the obtained results. Furthermore, in Section 5 a data fusion process is performed, on the data related to the wired accelerometers and to the QDaedalus total station. Finally, salient conclusions and remarks are outlined in closing Section 6.

\section{BRIEF INFORMATION ON THE BRIVIO BRIDGE}

The Brivio bridge is a three-span RC arch bridge located in the Lombardia region, northern Italy, near Milano. It crosses the Adda river at a height of about $8 \mathrm{~m}$ from water, between Brivio and Cisano Bergamasco, linking the two provinces of Lecco (LC) and Bergamo (BG). The construction of the bridge started in 1914 and was completed on May 1917 by the "Società Ferrobeton di Roma". The bridge was designed by Italian engineer G. Banfi on June 1912, while static tests were carried out in September $1916[7,13]$ and the bridge was opened to traffic on 1917.

The Brivio bridge is overall $130.8 \mathrm{~m}$ long and fully symmetric with respect to its mid longitudinal plane. A contemporary view of the bridge is pictured in Fig. 1.

The central span is $44 \mathrm{~m}$ long, while the lateral spans linked to the river banks are $43.4 \mathrm{~m}$ long. The road deck is $9.2 \mathrm{~m}$ wide; it hosts a double road line and two cantilever sidewalks, each $0.8 \mathrm{~m}$ wide. The deck of each span of the bridge comprises two main longitudinal beams $(0.45 \mathrm{~m} \times 1.00 \mathrm{~m})$ placed at a respective transverse distance of $8.60 \mathrm{~m}$. Between these, transverse beam connections $(0.30 \mathrm{~m} \times 0.75 \mathrm{~m})$ are provided at approximately every $2.30 \mathrm{~m}$. These transverse beams are further connected longitudinally by two further beams $(0.20 \mathrm{~m} \times 0.55 \mathrm{~m})$, placed symmetrically with respect to the vertical longitudinal plane of the bridge, at a distance 
of $1 \mathrm{~m}$. The resulting concrete frame is connected with an upper concrete slab, $0.15 \mathrm{~m}$ high that represents the bottom support of the road.

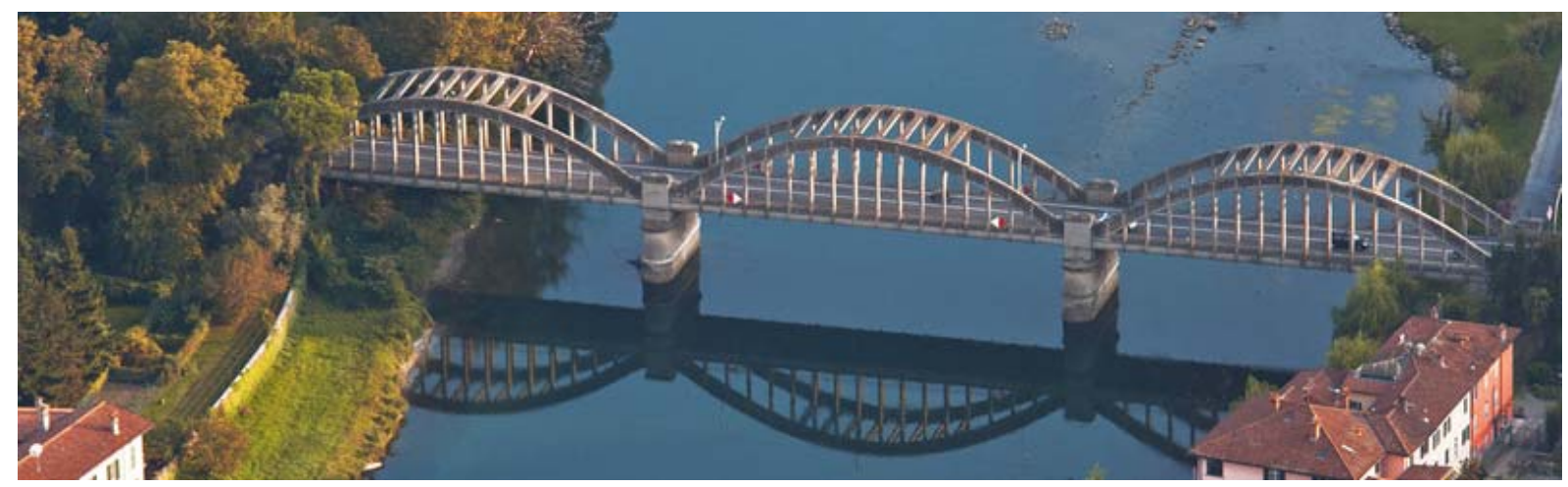

Fig. 1: Current view of the Brivio bridge (1917) crossing the Adda river. In the picture, the Brivio bank is located on the right.

The characteristic arches of the bridge structure display a span of $42.80 \mathrm{~m}$ and a rise of $8.00 \mathrm{~m}$. Each arch presents a rectangular cross section characterized by a constant width, equal to $0.60 \mathrm{~m}$, and a height variable from $1.25 \mathrm{~m}$ (on the middle) to $1.37 \mathrm{~m}$ (on the ends). The profile of the arch is symmetric with respect to the vertical axis at half span. The arches are linked to the road by means of sixteen hangers per each side, per each span, with rectangular cross section $0.32 \mathrm{~m}$ wide and $0.60 \mathrm{~m}$ high.

Two concrete piers constitute the intermediate supports of the deck into the river bed. The piers are tapered, with a maximum dimension at their base of $12.8 \mathrm{~m}$ along the transverse direction of the bridge and of $3.8 \mathrm{~m}$ along its longitudinal direction. Each pier rests on fortyeight reinforced concrete piles driven into the river bed, ranging from $13 \mathrm{~m}$ to $16 \mathrm{~m}$ of depth; each pile displays a square cross section of $0.35 \mathrm{~m}$ of side. Above both the intermediate piers and the abutments a reinforced concrete slab of $1 \mathrm{~m}$ of high is collocated.

Nowadays, after about 98 years of duty, the bridge is still in service and continuously subjected to intense traffic loading, characterized also by transit of heavy-weight vehicles, which induce high dynamical stresses and cyclic fatigue. Considering that the bridge still plays a very crucial role in the current local transportation network, queries about present and future structural performance of the bridge arise, while the bridge is approaching its hundred years of life-cycle.

\section{EXPERIMENTAL CAMPAIGN ON THE BRIVIO BRIDGE}

The experimental campaign has been performed on the Brivio bridge between June 11 and June 13, 2014. During these three days of measurements, four instrumentation systems were employed. Specifically, ten uniaxial wired piezoelectric accelerometers (AC), seven wireless sensors (WS), four QDaedalus system total stations (TS) and a laser scanner (LS) were used simultaneously for dynamic testing, according to different setups. The dynamic investigation of the bridge was carried out individually for each of the three spans. In particular, one span per day was monitored during the campaign. The AC, the WS and the TS were employed to target all spans, meanwhile the LS was used only for the first span of the bridge adjacent to the Brivio bank (Span 1, Day 1). In this paper, the main focus is placed on the measurements concerning the fist span, Span 1, of the bridge. The following Section 3.1 presents the setups adopted for each measurement system deployed during this experimental campaign. Section 3.2 reports a brief description of the employed instrumentation. 


\subsection{Measurement layout}

Based on the particular configuration of each instrumentation type, the setups of the measurements were planned so as to obtain signals that may easily be translated to corresponding points of the overall structure. The different layouts of the instrumentation adopted to record the response of Span 1 of the bridge are depicted in Figs. 3, 4. ACs, WSs and TSs were placed on the bridge according to two different setups (Setup 1 and Setup 2). A single setup was instead considered for the LS.

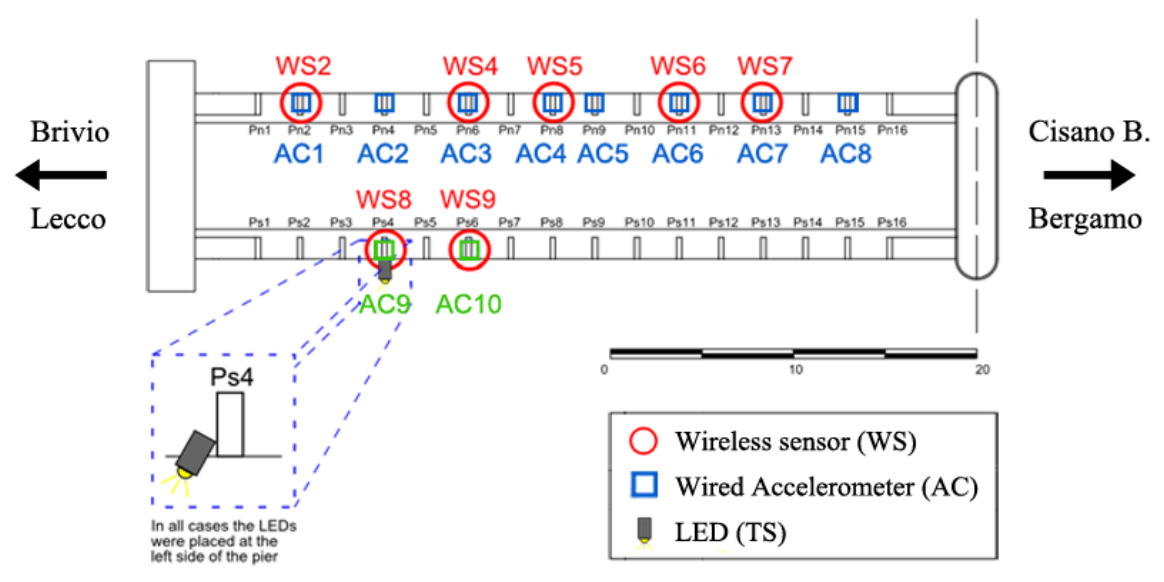

(a) Span 1 (Day 1) - Setup 1

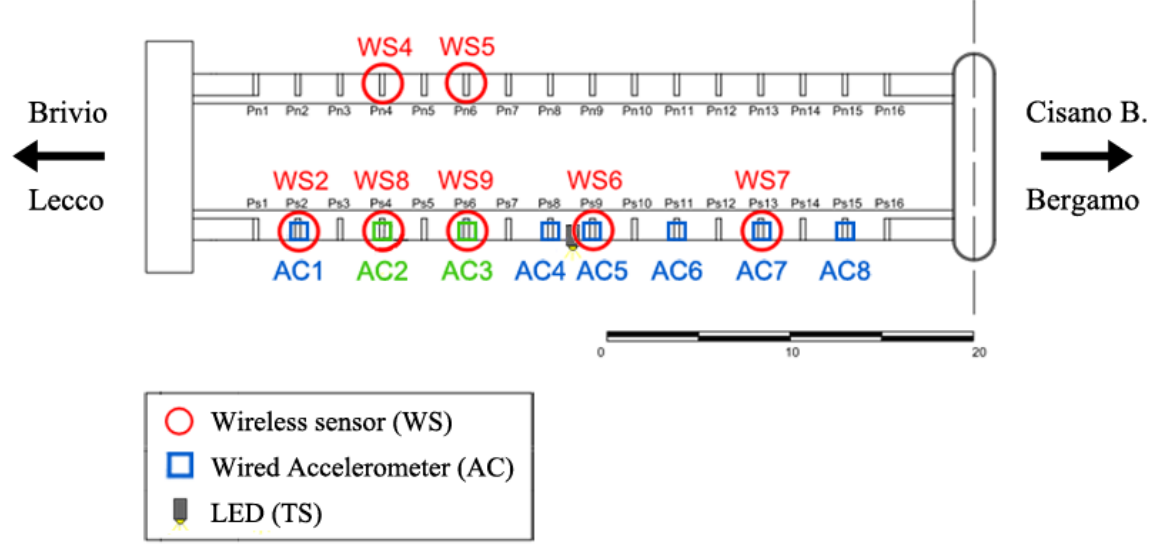

(b) Span 1 (Day 1) - Setup 2

Fig. 2: Setup 1 (a) and Setup 2 (b) of the wired accelerometers (AC), the wireless sensors (WS) and the LED used as targets for the total stations (TS) during the first day of measurements (Span 1).

As depicted in Fig. 2, the accelerations of the bridge pertaining to the AC system were measured on a total of eighteen selected points, considering two sensors as reference transducers (marked in green color in Fig. 2). Instead, the WS were disposed in seven points, selected as collocated to nodes of the tethered system, with the purpose of using the latter as a reference.

The TS were positioned on the bank of the Adda river on the Brivio side, at a distance from the bridge ranging from about $10 \mathrm{~m}$ to nearly $15 \mathrm{~m}$. Measurements were performed using two standard diodes (LED) as targets, per each setup, placed on the deck of the bridge according to the representations in Fig. 2. An additional setup was set also during the third day on the first span (Span 1), by adopting a LED target at midspan, i.e., between the AC4 and AC5 accelerometers (Fig. 2). Then, a total of two setups have been performed on the first span. 


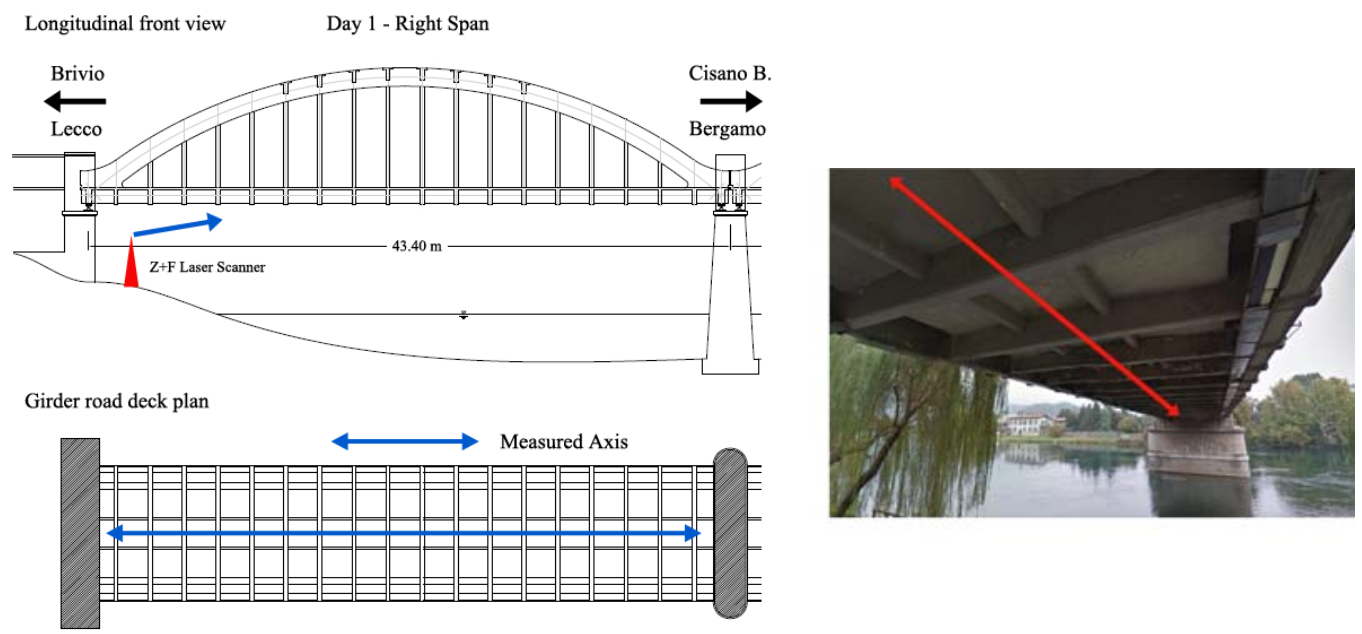

Fig. 3: Representation of the laser scanner setup targeting the span of the bridge from the Brivio bank.

The LS was positioned underneath the span of the bridge on the Brivio bank, where a narrow road passing under the structure is available for the inspection of the bridge from below. Such instrumentation was used in a "Profile Mode", which allows for dynamic measurements along a single axis. The system recorded the vertical displacements along the longitudinal axis of Span 1 of the bridge (Fig. 3).

During the experimental campaign the length of the recordings was variable, depending on the capacity of each sensor system. Details on the instrumentation are provided in the following Section 3.2.

\subsection{Description of the employed measurement systems}

In the following a brief description of each sensor system used during the experimental campaign is reported. A representation of the instrumentation is depicted in Fig. 4.
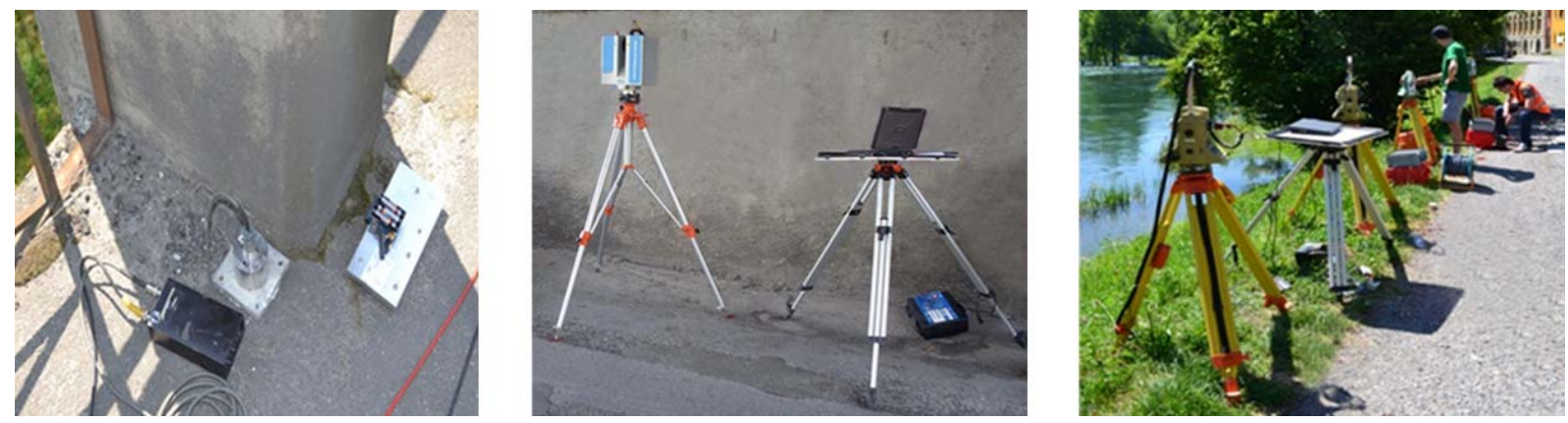

Fig. 4: Pictures of the adopted instrumentation. From the left: wireless sensors (WS) together with wired accelerometers (AC), laser scanner (LS), and Total Stations (TS).

\subsubsection{Wired accelerometers (AC)}

Wired accelerometers (AC) were provided by PoliMI (VibLab). They were used on the bridge in an integrated system, composed of: (a) a 24-channel data acquisition system, consisting of 6 NI 9234 4-channel dynamic signal acquisition modules (24-bit resolution, $102 \mathrm{~dB}$ dynamic range and anti-aliasing filters), interfaced to a remote PC and to its storage unit; (b) uniaxial WR 731A piezoelectric accelerometers on the roadway deck; each WR 731A sensor, capable of measuring accelerations up to $0.5 \mathrm{~g}$ with a sensitivity of $10 \mathrm{~V} / \mathrm{g}$, was connected with a short cable $(1 \mathrm{~m})$ to a WR P31 power unit/amplifier [2]. 


\subsubsection{Wireless sensors (WS)}

Wireless Sensors (WS) employed on the bridge have been supplied by the Chair of Structural Mechanics, at the Department of Civil, Enviromental and Geomatic Engineering of ETH Zürich. Each sensor is composed by an Imote2 Platform, furnished with an additional ITS400 measurement board. The sensor board is equipped with: (a) a 3D MEMS accelerometer LIS3LV02DQ (by STMicroelectronics); the accelerometer provides a 12bit resolution of a $2 \mathrm{~g}$ signal in all three spatial directions; (b) a CPU Intel XScala PXA27x processor, capable of operating in a vast frequency range, namely from $13 \mathrm{MHz}$ to $416 \mathrm{MHz}$; (c) a power management chip - Dialog DA9030; (d) an embedded wireless radio chip - CC2420. With a proper antenna device, this enables wireless communication in a range up to $100 \mathrm{~m}$ in the line of sight [8].

\subsubsection{Laser scanner (LS)}

A Z+F IMAGER ${ }^{\circledR}$ 5006i Laser Scanner, based upon the spot Z+F Laser Measurement System LARA, was provided by the Institute of Geodesy and Photogrammetry (IGP), ETH Zürich, to perform profile scans of the Brivio bridge. It consists of an industrial imaging 3D laser measurement system, which is generally employed in the fields of digital planning of factories, industrial plants, protection of historical monuments, landscape, virtual reality and so on. The noise of the system at a $10 \mathrm{~m}$ range lies in the $0.4 \mathrm{~mm}$ RMS (reflectivity $100 \%$, white) to $1.2 \mathrm{~mm}$ RMS (reflectivity 10\%, black) range, making the employment of the instrument worth attempting on the Brivio bridge.

\subsubsection{Total stations (TS)}

The QDaedalus measuring system, herein referred to as Total Station (TS) was designed and developed at the Geodesy and Geodynamics Lab (GGL) of ETH Zürich. The system is called QDaedalus and is able to perform fully-automated high-precision digital angle measurements. It was designed primarily for automated on-line astro-geodetic measurements [4], but its use demonstrated promising potentialities also in other disciplines, such as in real-time deformation and vibration analysis [4], as further enquired here. QDaedalus consists of (a) a small charge-coupled device camera which can easily be clipped to a Total Station, instead of an ordinary eye-piece, (b) a pluggable front lens, (c) a GNSS receiver, and (d) a dedicated software for steering, imaging and on-line processing. The system was employed on the bridge with TCA 1800 and TPS 1200 Total Stations from Leica Geosystems. Moreover, highprecision time-tagged measurements were possible by means of a GNSS receiver.

\section{DYNAMIC TESTS AND MODAL DYNAMIC IDENTIFICATION OF THE BRIDGE}

Sections 4.1-4.4 report the modal estimates obtained from frequency based identification procedures, based on the response data acquired by each instrumentation system employed on the bridge.

\subsection{Wired Accelerometers (AC) tests}

During the experimental campaign, two time series of $3600 \mathrm{~s}$ were recorded per setup (Fig. 2), with a sampling rate of $200 \mathrm{~Hz}$. Then, low-pass filtering and decimation were applied to the recordings before modal identification, by reducing the frequency sampling from $200 \mathrm{~Hz}$ down to $25 \mathrm{~Hz}$. 


\subsubsection{Modal identification based on AC data}

The output-only modal identification was carried out by using both the Frequency Domain Decomposition (FDD) [3] and the data-driven Stochastic Subspace Identification (SSIdata) [9] methods available in the commercial software ARTeMIS [15].

The results of the detailed AVT performed with the conventional high-sensitivity accelerometers are extensively reported in the work [6]. Herein, the modal frequencies obtained from the identification procedures are summarized in Table 1.

\begin{tabular}{cccccccccc}
\hline Modes & 1 & 2 & 3 & 4 & 5 & 6 & 7 & 8 & 9 \\
\hline$f_{\text {FDD }}[\mathrm{Hz}]$ & 3.564 & 3.857 & 6.018 & 7.178 & 7.690 & 9.009 & 11.377 & 13.086 & 17.017 \\
$f_{\text {SSI }}[\mathrm{Hz}]$ & 3.449 & 3.887 & 5.968 & 7.146 & 7.592 & 8.928 & 11.390 & 13.040 & 16.990 \\
\hline$\Delta f[\%]$ & 3.23 & 0.78 & 0.83 & 0.45 & 1.27 & 0.90 & 0.11 & 0.35 & 0.16 \\
\hline
\end{tabular}

Table 1: Identified frequencies $f_{\mathrm{i}}[\mathrm{Hz}]$ through FDD method $\left(f_{\mathrm{FDD}}\right)$ and SSI method $\left(f_{\mathrm{sSI}}\right)$, first span, wired accelerometers (AC).

The estimates coming from the application of the FDD algorithm, hereafter referred to as $f_{\mathrm{AC}}$, are assumed as the identification target for the further instrumentation systems.

\subsection{Wireless Sensors (WS) tests}

During the experimental campaign, a total of nine tests have been performed on the first span, characterized by a variable number of sensors, varying from three to five in a single test. The sensors recorded time series of $60 \mathrm{~s}$ with a sampling rate of $32 \mathrm{~Hz}$ (for a total of 1920 samples). Low-pass filtering at $14 \mathrm{~Hz}$ was performed on the acquired data. The low sampling frequency and short duration of the signals is due to the characteristics of the adopted instrumentation.

\subsubsection{Modal identification based on WS data}

Initially, attempts with a classical FDD approach [3] have been performed. The short duration of the recordings allows for the averaging of a maximum of 512-points Hanning smoothing windows with $66.7 \%$ overlapping, resulting in poorly-defined PSD matrices, with low frequency resolution equal to $0.0313 \mathrm{~Hz}$. Further, the SV graphs presented noisy and unclear curves, leading to very difficult modal peak detection. Tests were also performed adopting a zero-padding of at least $300 \mathrm{~s}$ at the end of the original signals to artificially increment their length [10]. In this way, it was possible to adopt 1024-points and 2048-points Hanning smoothing windows. The obtained results however remained rather poor in this case as well.

Next, a second approach relying on a refined FDD (rFDD) method [10,11] was adopted, seeking for better estimates. The rFDD algorithm implements in a self-contained and efficient framework the Wiener-Khinchin theorem [1], adopting the 2048-point transform of a biased and untrended correlation matrix for the PSD matrix estimate. This method, especially intended for very short structural recordings (including e.g. seismic response recordings) in which the averaging implies loss of considerable information, returns clearer and well-defined modal peaks, with respect to those achievable from the Welch approach [16] in the PSD matrix computation. In this case, the frequency resolution became $0.0156 \mathrm{~Hz}$.

Despite the lower number of sensors, the symmetries and the geometrical properties of the structure, along with some inspections using MAC and MPC indexes (in their Auto- formulations) allowed to simulate the components of the mode shapes also were channels did not 
cover the deck. This indicates that WSNs may be adopted as a suitable technology for the instrumentation of bridge systems, combining fast deployment and low costs with sufficient accuracy.

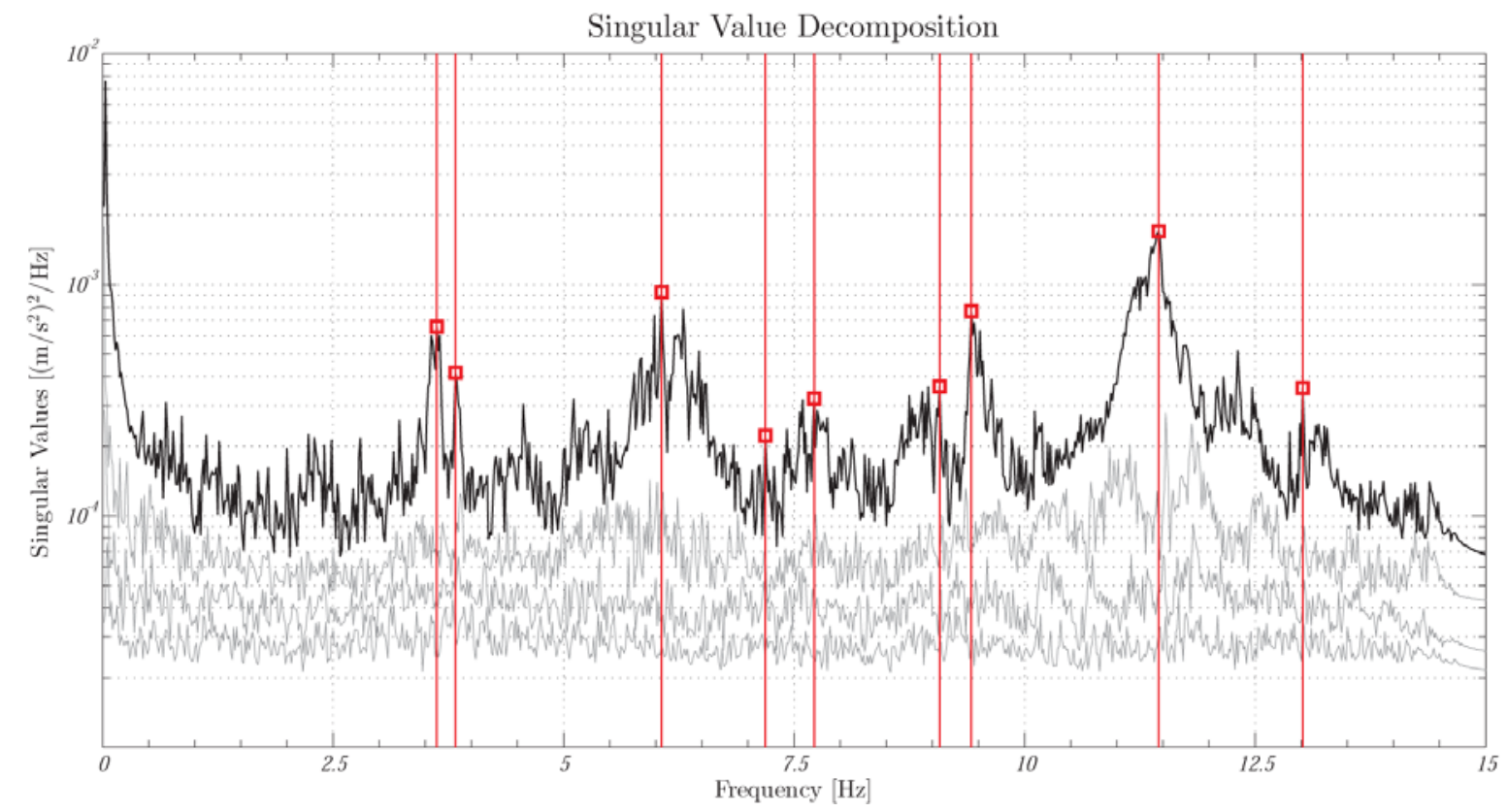

Fig. 5: Singular Value Decomposition and peak-picking technique for Test 1 (Setup 1), Wireless Sensors (WS) measurements.

\begin{tabular}{|c|c|c|c|c|c|c|c|c|c|c|}
\hline Setup & Tests & 1 & 2 & 3 & 4 & 5 & 6 & 7 & 8 & 9 \\
\hline \multirow{6}{*}{1} & 1 & 3.625 & 3.828 & 6.063 & 7.188 & 7.719 & 9.078 & 9.422 & 11.453 & 13.016 \\
\hline & 2 & 3.609 & 3.813 & 5.828 & 7.266 & 7.688 & 9.047 & 9.391 & 11.406 & 13.359 \\
\hline & 3 & 3.609 & 3.813 & 5.828 & 7.266 & 7.688 & 9.047 & 9.391 & 11.938 & 13.031 \\
\hline & Mean & 3.614 & 3.818 & 5.906 & 7.240 & 7.698 & 9.057 & 9.401 & 11.599 & 13.135 \\
\hline & STD & 0.009 & 0.009 & 0.136 & 0.045 & 0.018 & 0.018 & 0.018 & 0.295 & 0.194 \\
\hline & $\Delta f_{\mathrm{AC}}[\%]$ & 1.41 & 1.01 & 1.86 & 0.86 & 0.11 & 0.54 & - & 1.95 & 0.38 \\
\hline \multirow{9}{*}{2} & 4 & 3.641 & 3.844 & 6.109 & 7.234 & 7.656 & 9.047 & 9.313 & 11.406 & 13.563 \\
\hline & 5 & 3.281 & 3.516 & 5.969 & 7.281 & 7.906 & 9.031 & 9.922 & 11.406 & 13.520 \\
\hline & 6 & 3.516 & 3.719 & 5.969 & 7.188 & 7.797 & 9.078 & 9.641 & 11.203 & 13.188 \\
\hline & 7 & 3.547 & 3.922 & 6.172 & 7.234 & 7.703 & 8.953 & 9.531 & 11.328 & 12.970 \\
\hline & 8 & 3.641 & 3.922 & 6.109 & 7.141 & 7.797 & 9.281 & 9.766 & 11.453 & 13.188 \\
\hline & 9 & 3.578 & 3.859 & 6.172 & 7.234 & 7.875 & 8.984 & 9.469 & 11.530 & 12.970 \\
\hline & Mean & 3.534 & 3.797 & 6.083 & 7.219 & 7.789 & 9.062 & 9.607 & 11.388 & 13.233 \\
\hline & STD & 0.134 & 0.156 & 0.093 & 0.048 & 0.096 & 0.116 & 0.218 & 0.112 & 0.258 \\
\hline & $\Delta f_{\mathrm{AC}}[\%]$ & 0.84 & 1.56 & 1.09 & 0.57 & 1.29 & 0.59 & - & 0.09 & 1.12 \\
\hline
\end{tabular}

Table 2: Identified frequencies $f_{\mathrm{i}}[\mathrm{Hz}]$, mean value, standard deviation and discrepancy $\Delta f_{\mathrm{AC}}$, first span, wireless sensors (WS), for the two setups illustrated in Fig. 2. 
Fig. 5 shows the SVD obtained by the application of the rFDD technique to the data collected during the first test performed on the bridge according to Setup 1. In this test, four sensors were used, in particular devices WS4, WS5, WS6 and WS8, as depicted in Fig. 2. Singular Value peaks were identified until $14 \mathrm{~Hz}$, due to the sampling rate of the signal. The frequencies arising from all of the nine tests performed on the bridge are reported in Table 2, along with the mean value and the standard deviation of the results and the discrepancy $\Delta f_{\mathrm{AC}}$ between the mean values and the reference frequencies refer to the AC (Table 1), for each setup.

Rather limited standard deviations were obtained from the overall tests performed on the bridge. During the experimental campaign, the capabilities of the WSN were also tested at various transmission rates, namely $45 \%, 35 \%$ and $25 \%$. The data from such tests were analyzed through the rFDD method, relying on 2048-point transform of a biased and untrended correlation matrix for the PSD matrix estimate. Table 3 reports the obtained results, in terms of the discrepancy $\Delta f_{\mathrm{AC}}$ between the mean values of the identified frequencies calculated for each setup and the $f_{\mathrm{AC}}$ reference values, for each transmission rate. A quite clear SV representation was obtained up to $8 \mathrm{~Hz}$, in particular for the peaks at around $3.9 \mathrm{~Hz}$ (Mode 2), $6 \mathrm{~Hz}$ (Mode 3) and $7.7 \mathrm{~Hz}$ (Mode 5).

\begin{tabular}{lcccccc}
\hline Setup & Mests & 1 & 2 & 3 & 4 & 5 \\
\hline \multirow{2}{*}{$\mathbf{1}$} & $f_{\mathrm{AC}}[\mathrm{Hz}]$ & 3.564 & 3.857 & 6.018 & 7.178 & 7.690 \\
\hline & $\mathrm{r} 45$ & $3.40 \%$ & $0.88 \%$ & $3.42 \%$ & $0.35 \%$ & $0.72 \%$ \\
\cline { 2 - 7 } & $\mathrm{r} 35$ & $0.04 \%$ & $0.34 \%$ & $0.13 \%$ & $2.67 \%$ & $1.80 \%$ \\
\hline \multirow{2}{*}{$\mathbf{2}$} & $\mathrm{r} 25$ & $4.41 \%$ & $3.31 \%$ & $1.52 \%$ & $1.10 \%$ & $0.58 \%$ \\
\cline { 2 - 7 } & $\mathrm{r} 45$ & $2.01 \%$ & $0.81 \%$ & $1.26 \%$ & $0.13 \%$ & $1.19 \%$ \\
\cline { 2 - 7 } & $\mathrm{r} 35$ & $2.74 \%$ & $2.57 \%$ & $2.43 \%$ & $0.05 \%$ & $0.51 \%$ \\
\hline
\end{tabular}

Table 3: Discrepancy $\Delta f_{\mathrm{AC}}$ of the results obtained considering different WSN transmission rates, first span, for the two setups illustrated in Fig. 2.Results r45, r35 and r25 refer to the rate 45\%, 35\% and 25\% respectively.

At present, the sensors employed during this experimental campaign are the subject of a research project at ETH Zürich, aimed at extending the applicability of currently-available WSN systems, by combining recently-surfaced energy harvesting methods with up-to-date data compression methods [8].

\subsection{Laser Scanner (LS) tests}

The $\mathrm{Z}+\mathrm{F}$ Imager measures an angle from the rotary motor of the device and a distance corresponding to each angle with its laser. It is worth to note that a fixed angle of the rotary motor would not lead to track exactly the same point of a vibrating structure. Thus, the concept of "y-bins" was used to create clusters of points along the y-axis. In each bin, the mean value of vertical-direction/displacement-history were obtained and used for the frequency analysis presented in this work. The system provides readings in a .zfs file format. The original data files were converted into ASCII format (.ptx) and then treated through a MATLAB script. 
During the experimental campaign the resolution (subsampling) of the LS was chosen as 10000 pixels/360 degrees and the sampling frequency was equal to $25 \mathrm{~Hz}$, for recorded time series of $1100 \mathrm{~s}$. Low-pass filtering at $10 \mathrm{~Hz}$ was performed on the recorded data. A total of five tests have been performed on the first span; for each test, the displacement histories of the road deck from underneath were extracted for the clusters of points (y-bins) located in correspondence of all wired accelerometers (Fig. 2).

\subsubsection{Modal identification based on LS data}

For modal identification purposes, only results arising from two selected points, i.e., located on the scanned profile and corresponding to the positions of the wired accelerometers used as reference devices (in green color in Fig. 2) are presented. Hereafter, the measurements relating to these two points are referred to as Setup 1 (point in correspondence of AC9/AC2 in Fig. 2) and Setup 2 (point in correspondence of AC10/AC3 in Fig. 2).

For each displacement/time record, an Auto-PSD estimate was computed by using the Welch Modified Periodogram method, set with 2048-points Hanning smoothing windows and $66.7 \%$ overlapping. Then, peak-picking has been performed over the achieved PSD, in order to obtain first frequency estimates, via the Basic Frequency Domain approach (BFD) [1]. The obtained frequency resolution was $0.0122 \mathrm{~Hz}$.

Fig. 6 shows the PSD obtained by the application of the BFD technique to the data collected during the fourth test of the second setup. In the figure, the frequencies obtained through a peak-picking procedure are highlighted by means of vertical red lines. Table 3 reports the frequencies extracted through such procedure for all the measurements performed on the first span. The listed values pertain to the range of frequencies identified through the acceleration signals (AC). The values of the discrepancy $\Delta f_{\mathrm{AC}}$ between the mean values and the reference frequencies refer to the AC (Table 1) were also calculated when possible.

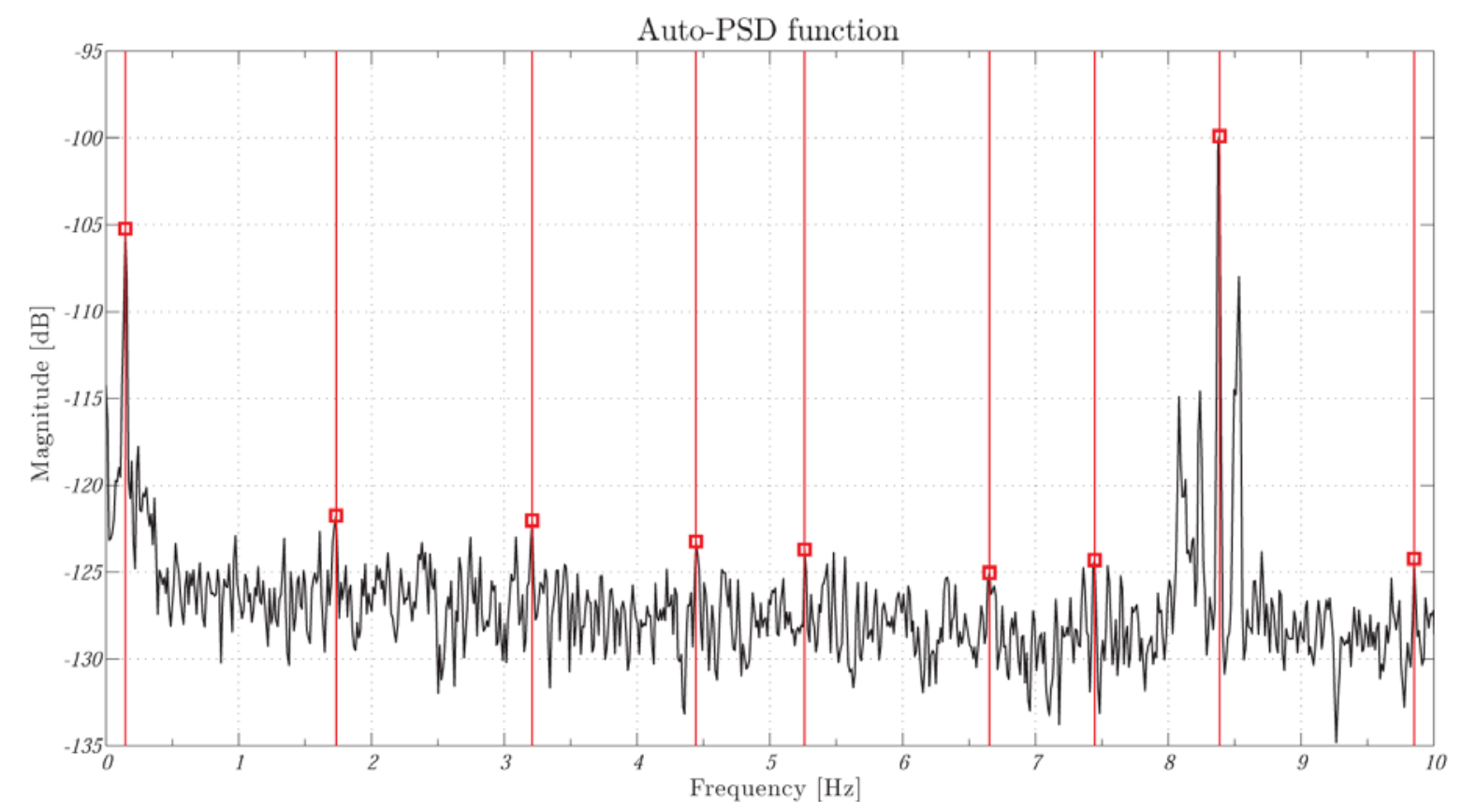

Fig. 6: Welch Auto-PSD estimate and peak-picking technique for Test 4 (Setup 2), Laser Scanner (LS) measurements. 
R. Ferrari, F. Pioldi, E. Rizzi, C. Gentile, E. Chatzi, R. Klis, E. Serantoni, A. Wieser

\begin{tabular}{|c|c|c|c|c|c|c|c|c|}
\hline Setup & Tests & 1 & 2 & 3 & 4 & 5 & 6 & 7 \\
\hline \multirow{8}{*}{1} & 1 & 3.540 & 4.431 & 5.322 & 6.226 & 7.446 & - & 9.436 \\
\hline & 2 & 3.210 & 4.895 & 5.334 & 6.055 & 7.031 & 8.374 & - \\
\hline & 3 & 3.210 & 4.553 & 5.652 & 6.445 & 7.300 & 8.374 & 9.412 \\
\hline & 4 & 3.613 & 4.176 & 5.469 & 6.201 & 7.056 & 8.386 & 9.607 \\
\hline & 5 & 3.589 & 4.602 & 5.371 & 6.421 & 7.666 & 8.374 & - \\
\hline & Mean & 3.432 & 4.531 & 5.430 & 6.270 & 7.300 & 8.377 & 9.485 \\
\hline & STD & 0.205 & 0.262 & 0.137 & 0.163 & 0.268 & 0.006 & 0.106 \\
\hline & $\Delta f_{\mathrm{AC}}[\%]$ & 3.69 & - & - & 4.18 & 5.07 & - & 5.28 \\
\hline \multirow{8}{*}{. } & 1 & 3.162 & 4.199 & - & 6.604 & 7.336 & 8.374 & 9.094 \\
\hline & 2 & 3.162 & 4.211 & 5.115 & 6.655 & 7.520 & 8.374 & 9.900 \\
\hline & 3 & 3.711 & 4.883 & 5.847 & - & 7.544 & 8.374 & 9.460 \\
\hline & 4 & 3.210 & 4.443 & 5.261 & 6.653 & 7.446 & 8.386 & 9.851 \\
\hline & 5 & 3.482 & - & 5.542 & 6.522 & - & 8.374 & - \\
\hline & Mean & 3.345 & 4.434 & 5.441 & 6.609 & 7.462 & 8.376 & 9.576 \\
\hline & STD & 0.244 & 0.320 & 0.323 & 0.062 & 0.093 & 0.005 & 0.377 \\
\hline & $\Delta f_{\mathrm{AC}}[\%]$ & 6.13 & - & - & 9.81 & 2.97 & - & 6.30 \\
\hline
\end{tabular}

Table 3: Identified frequencies $f_{\mathrm{i}}[\mathrm{Hz}]$, mean value, standard deviation and discrepancy $\Delta f_{\mathrm{AC}}$, first span, $\mathrm{Z}+\mathrm{F}$ Laser Scanner (LS), for the two setups illustrated in Fig. 3.

In light of the obtained results, frequency estimates appear as more challenging with respect to the instrumentation options presented in the previous Sections 4.1-4.2. After all, it is worth to note that in this case the identification procedure relies solely on a simplified peakpicking procedure. Surely, other methods, possibly formulated in the time domain, need be considered.

Original attempts with the rFDD approach [10,11,12] are currently under target using the LS data. The aim of such post-processing analysis is that of considering selected points as acquisition channels. This should allow to identify the mode shapes of the bridge, in addition to the modal frequencies.

Observing the results obtained from the LS, few considerations arise concerning: (a) the manifested peak at about $8 \mathrm{~Hz}$; (b) the peak at low frequency ( $<0.5 \mathrm{~Hz})$. Regarding (a), some independent and dedicated measurements performed at ETH Zurich permitted to associate such peak at only mechanical functioning of the instrument. Steps to resolve this issue are the object of current investigations.

The authors feel that the Laser Scanner offers high potential for structural monitoring purposes for large scale structures, albeit its use in the field of the SHM still limited and obviously demands further exploration with respect to appropriate post-processing methodologies.

\subsection{Total Stations (TS) tests}

The first setup, consisting of two tests, had a sampling rate of $60 \mathrm{~Hz}$, with lengths of $2200 \mathrm{~s}$ and $1200 \mathrm{~s}$, respectively. The second setup, made by a single test (performed on the third day), had a sampling rate of $30 \mathrm{~Hz}$, with a length of $4200 \mathrm{~s}$. Low-pass filtering at $15 \mathrm{~Hz}$ was chosen for the first setup, while the cutoff was set at $10 \mathrm{~Hz}$ for the second setup. Also, a detrending of the obtained displacements was performed. 


\subsubsection{Modal identification based on TS data}

The Welch Modified Periodogram method was adopted for the Auto-PSD matrix estimate, set with 1024-points Hanning smoothing windows and 66.7\% overlapping. Afterwards, peakpicking was performed over the calculated PSD, in order to obtain first frequency estimates, via a BFD approach [1]. The obtained frequency resolution was $0.0293 \mathrm{~Hz}$. Tests were also performed without filtering, without detrending, and with 512- or 2048-points Hanning smoothing windows.

The obtained Auto-PSD of the second test (Setup 1) is represented in Fig. 7, where a total of nine peaks extracted through a peak-picking procedure are marked with vertical red lines. Table 4 reports the estimated frequencies of all the setups adopted on the first span, within the range of frequencies identified from the $\mathrm{AC}$, along with the values of the discrepancy $\Delta f_{\mathrm{AC}}$ between the mean values and the reference frequencies (Table 1).

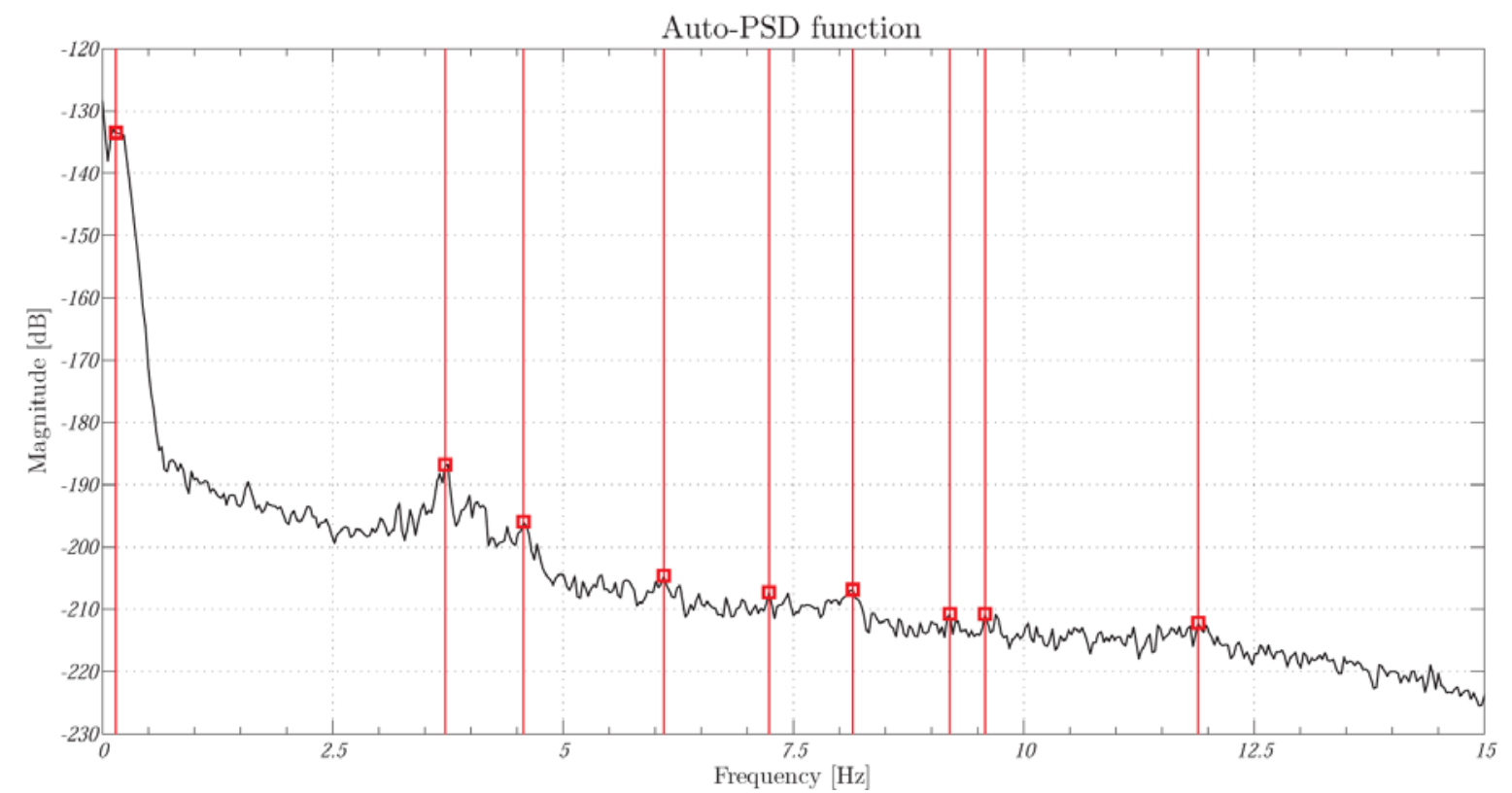

Fig. 7: Welch Auto-PSD estimate and peak-picking technique for Test 2 (Setup 1), QDaedalus Total Station (TS) measurements.

\begin{tabular}{|c|c|c|c|c|c|c|c|c|c|}
\hline Setup & $\begin{array}{ll} & \text { Modes } \\
\text { Tests } & \end{array}$ & 1 & 2 & 3 & 4 & 5 & 6 & 7 & 8 \\
\hline \multirow{5}{*}{1} & 1 & 3.369 & 4.424 & 6.211 & 7.119 & 7.939 & 9.551 & 10.459 & 11.777 \\
\hline & 2 & 3.721 & 4.570 & 6.094 & 7.236 & 8.145 & 9.199 & 9.580 & 11.895 \\
\hline & Mean & 3.545 & 4.497 & 6.153 & 7.178 & 8.042 & 9.375 & 10.020 & 11.836 \\
\hline & STD & 0.249 & 0.103 & 0.083 & 0.083 & 0.146 & 0.249 & 0.622 & 0.083 \\
\hline & $\Delta f_{\mathrm{AC}}[\%]$ & - & 16.59 & 2.23 & 0.01 & 4.58 & 4.06 & - & 4.03 \\
\hline \multirow{2}{*}{2} & 3 & 3.501 & 4.072 & 5.918 & 7.280 & 7.969 & 9.009 & 9.536 & - \\
\hline & $\Delta f_{\mathrm{AC}}[\%]$ & 1.77 & 5.57 & 1.66 & 1.42 & 3.63 & 0.01 & - & - \\
\hline
\end{tabular}

Table 4: Identified frequencies $f_{\mathrm{i}}[\mathrm{Hz}]$, mean value, standard deviation and discrepancy $\Delta f_{\mathrm{AC}}$, first span, QDaedalus Total Stations (TS), for the two setups illustrated in Fig. 2. 
Also in this case relatively high standard deviations were calculated from the obtained results. Despite this, the Auto-PSD representation appears improved with respect to that of the LS system. In fact, from Fig. 7 it is possible to observe amplitude amplifications in the frequency range corresponding to the first vibration modes of the bridge $(3.5-3.9 \mathrm{~Hz})$. In order to improve results, a data fusion between the displacements from the QDaedalus and the reference accelerations (from the AC devices) is operated next.

\section{DATA FUSION VIA KALMAN FILTERING}

In this section, the use of the reference acceleration measurements (AC) is presented in order to complement the noisy dynamic displacement measurements collected during the experimental campaign on the bridge. In particular, a fusion between the accelerations measured through the standard wired accelerometers (AC) and the displacements obtained from the total stations (TS) was performed using a Kalman filtering technique. The results herein reported refer to the displacements obtained via the QDaedalus system during the second test (Setup 1).

The first step towards this goal was that of aligning the two signals, which were originally not perfectly synchronized. This was not a trivial task, due to (a) the different nature of the records (displacements vs. accelerations); (b) the different content of noise in the measurements; (c) the sampling, both in terms of its regularity and order of magnitude. The signals were aligned through a MATLAB script, which operates on the basis of determining the time leads or lags of two strings of data, locating the maximum value of their cross-correlation. Primarily, the two signals were detrended and filtered. A low-pass filter designed through a Chebyshev Type II method was applied to the accelerations at $25 \mathrm{~Hz}$. Then, due to the inability of the AC devices to register the frequency content below $0.5 \mathrm{~Hz}$, a high-pass filter was operated on both the AC accelerations and the QDaedalus displacements. The alignment procedure was operated considering displacements. Therefore, an integration procedure was operated on the AC accelerations. Appropriate filtering was performed during the integration steps. Then, a re-sampling of the signal using a polyphase filter implementation was operated on the obtained displacements, to reduce sampling from $200 \mathrm{~Hz}$ down to $50 \mathrm{~Hz}$, as for the available measurements from the QDaedalus. A cross-correlation calculation is then carried out for specifying the lag between the signals.

The results reported herein refer to an analysis conducted considering a time window about 10 minutes duration. Since accelerations and displacements were measured at different sampling rates, namely $200 \mathrm{~Hz}$ and $50 \mathrm{~Hz}$ respectively, a multi-rate Kalman filter was involved in the data fusion procedure.

The Kalman filter was considered by assuming the following standard state-space model:

$$
\begin{aligned}
& \dot{\mathbf{x}}(t)=\left[\begin{array}{c}
\dot{x}(t) \\
\ddot{x}(t)
\end{array}\right]=\left[\begin{array}{ll}
0 & 1 \\
0 & 0
\end{array}\right]\left[\begin{array}{c}
x(t) \\
\dot{x}(t)
\end{array}\right]+\left[\begin{array}{c}
0 \\
\ddot{x}_{m}(t)
\end{array}\right]+\mathbf{v}(t) \\
& \mathrm{y}(t)=x_{m}(t)=\left[\begin{array}{ll}
1 & 0
\end{array}\right]\left[\begin{array}{l}
x(t) \\
\dot{x}(t)
\end{array}\right]+\eta(t)
\end{aligned}
$$

where $\mathbf{x}(t)$ is the system state vector comprising the displacement $\mathbf{x}(t)$ and velocity $\dot{x}(t)$, $\mathbf{v}(t)$ the process noise data structure (Gaussian), $y(t)$ the noisy observation of the system (herein the QDaedalus displacements $\left.x_{m}(t)\right), \eta(t)$ the observation noise data structure (Gaussian), $\ddot{x}_{m}(t)$ denotes the exogenous input to the state transition function, which effectively here pertains to the measured acceleration. The interested reader may refer to [14] on 
further details on the implementation of the Multi-Rate Kalman Filter on the above-mentioned system.

The following figures illustrate the results obtained from the implemented data fusion procedure.

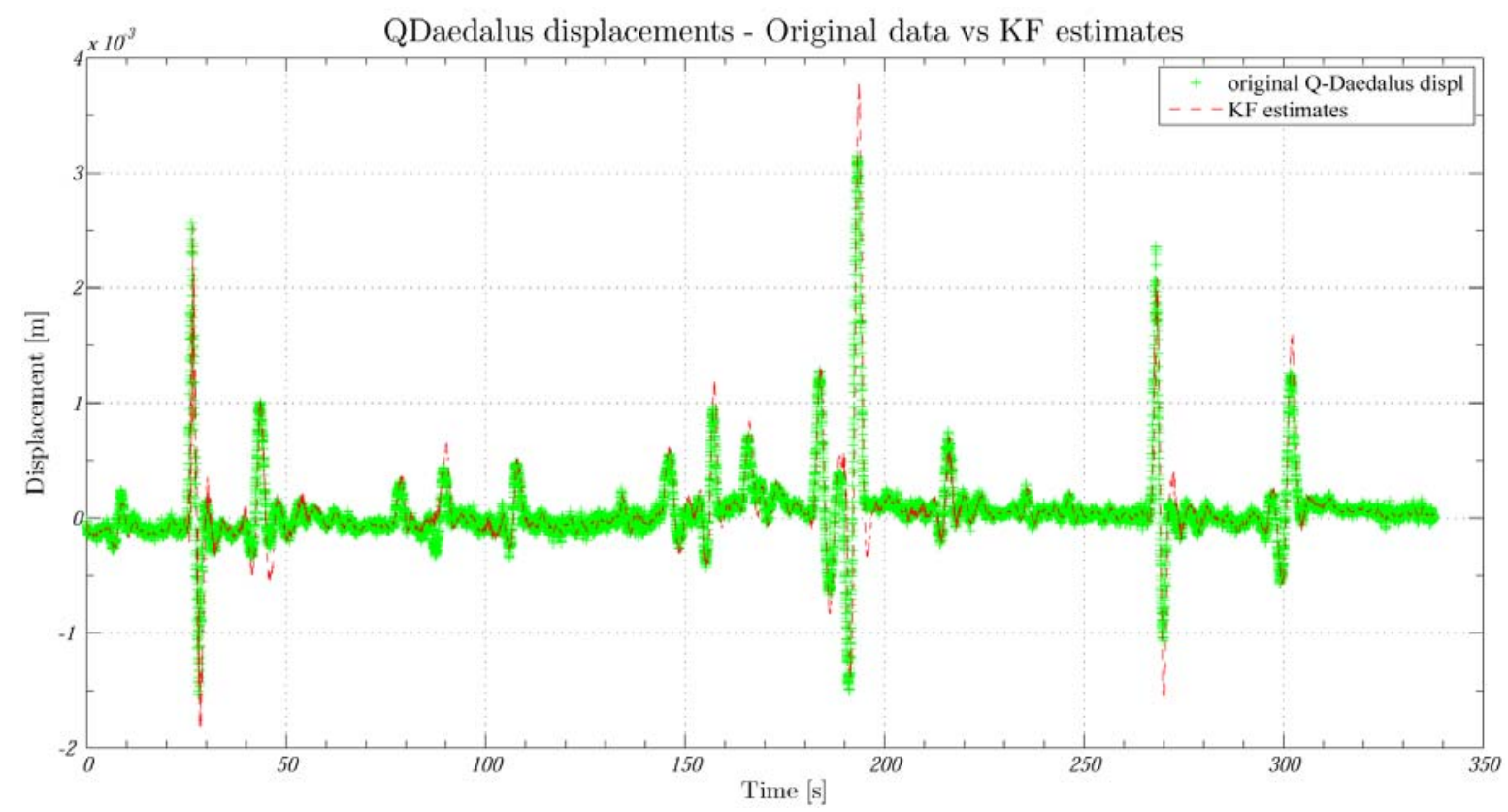

Fig. 8: QDaedalus displacements before and after the Multi-Rate Kalman filter application.

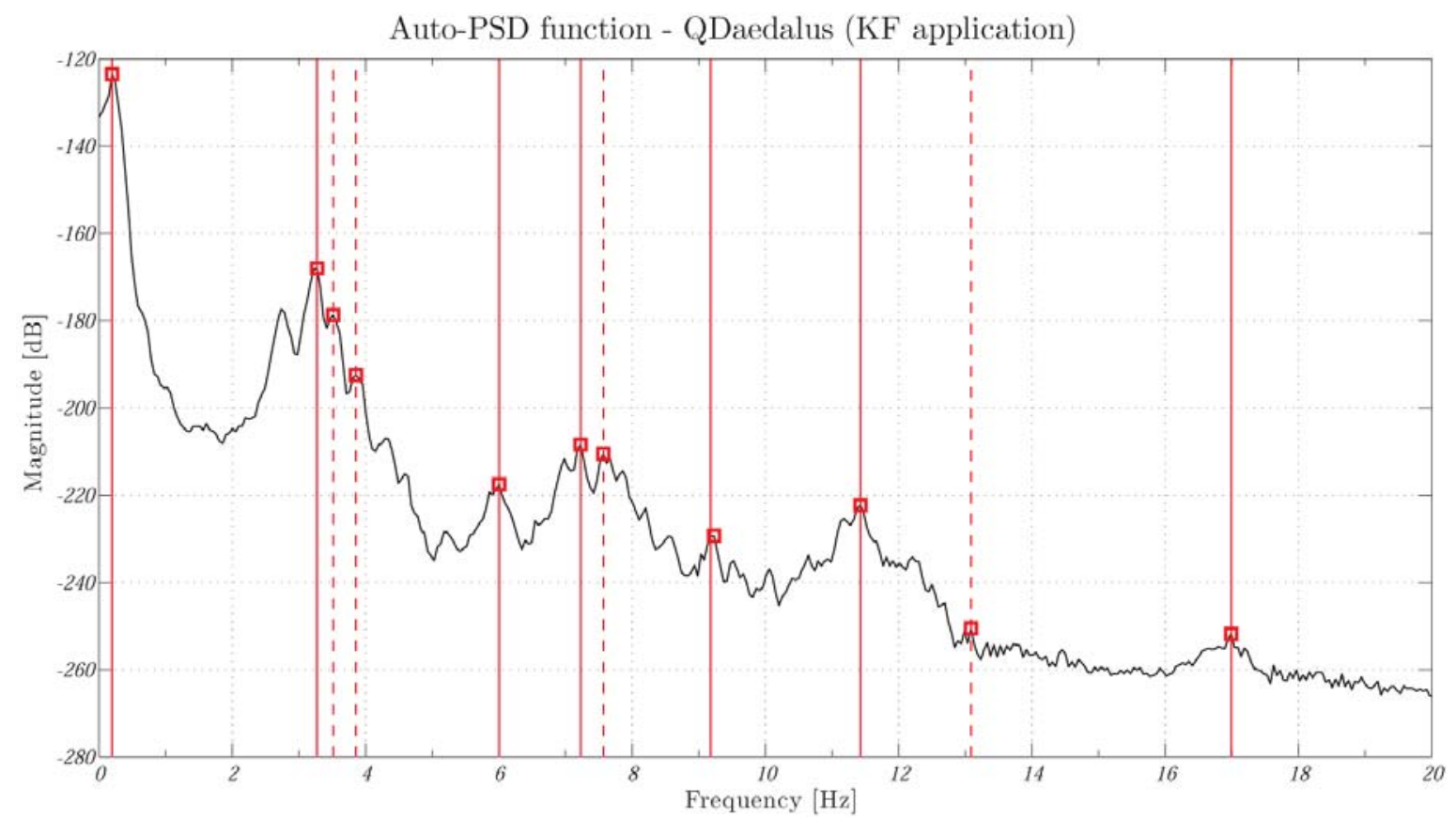

Fig. 9: PSD estimate referred to the measured QDaedalus displacements after data fusion.

Through the Kalman filter based fusion it was possible to obtain refined displacement estimates of improved accuracy. Fig. 8 shows the QDaedalus displacements before and after the fusion procedure. The quality of the obtained results is then clearly demonstrated in Fig. 9 where the Auto-PSD of the signal obtained after the data fusion is represented. The frequen- 
cies identified through an automatic peak-picking procedure are marked with vertical red lines; dashed lines mark instead the peaks that appear around the frequencies identified from the reference devices (AC) records. The representation of the Auto-PSD function, as visible in Fig. 9, appears improved in comparison to that obtained from the original displacements (depicted in the previous Fig. 7).

\section{CONCLUSIONS}

The experimental campaign conducted on the Brivio bridge provided the possibility to operate a comprehensive study regarding both the current modal dynamic identification of the structure and the reliability of the adopted types of instrumentation. A cross-comparison of the performance of standard wired accelerometers, wireless sensors and non-contact displacement measurement systems, i.e., a laser scanner and a QDaedalus total station solution, is carried out. The use and study of these novel instrumentation solutions may open up the way to several research scenarios, allowing to discover the true potential of these systems and to enrich or complement the information acquired via long-established instrumentation systems.

Initially, the present work analyzed the recordings arising from each individual instrumentation via system identification procedures, in order to assess their reciprocal reliability and consistency. Then, the focus was targeted on the use of a Kalman Filter approach for data fusion, able constructively merge of the data coming from displacement sensors and from standard wired accelerometers.

The results obtained from the different instrumentation systems employed on the bridge were compared in terms of identified structural modal properties (natural frequencies), through traditional Frequency Domain methods.

The output from wired accelerometers (AC) was taken here as a benchmark reference. Several vibration modes were identified in the frequency range up to $20 \mathrm{~Hz}$, giving rise to a well-defined set of modal parameters (see also companion work [6]).

The wireless MEMS accelerometers sensors (WS) demonstrated a high level of agreement with the results obtained from the AC, despite the limited length of the signals and their significant noise content, displaying rather small standard deviations among the various setups. This technology looks rather promising and is currently under investigation for possible further enhancement, by combining recently-surfaced energy harvesting methods and up-to-date data compression algorithms.

The obtained non-contact displacement measurements, i.e., those attained via the laser scanner and the Qdaedalus total stations, provided lower resolution results, but in agreement with outcomes from traditional AC recordings. The present considerations suggest that these techniques may be yet a bit "unripe", towards autonomous and independent modal identification, with respect to target estimates that may be achieved from standard AC system. However, the results obtained from a data fusion procedure carried out by means of a Multi-Rate Kalman Filter appear really encouraging, in showing such non-contact instrumentation as complementary systems to the standard AC solutions.

Studies to further improve the post-processing of the recordings of the newly investigated displacement measuring systems are in progress, in particular concerning the laser scanner solution. In parallel to the present study, the FE model updating of the developed FE models of the bridge is also on-going, with a first reporting of the outcomes provided in companion work [6]. 


\section{ACKNOWLEDGMENTS}

The authors thank very much the Province of Lecco, owner of the bridge, from allowing to perform the experimental tests; the cooperation of MSc A. Valsecchi (Director of the Bridge and Road Division, Province of Lecco) is gratefully acknowledged. The financial support by the University of Bergamo and by the Institute of Structural Engineering (IBK), ETH Zürich, within project ITALYR (Italian TALented Young Researchers) 2014, is also gratefully acknowledged. The authors would finally like to thank the Institute of Geodesy and Photogrammetry (IGP) and the Geodesy and Geodynamics Lab (GGL), ETH Zürich, for supplying the Z+F Laser Scanner and the Qdaedalus measurement systems.

\section{REFERENCES}

[1] J. Bendat, A. Piersol, Engineering Applications of Correlation and Spectral Analysis, 2nd Edition. Wiley, New York, 1993.

[2] F. Benedettini, C. Gentile, Operational modal testing and FE model tuning of a cablestayed bridge. Engineering Structures, 33(6), 2063-2073, 2011.

[3] R. Brincker, L. Zhang, P. Andersen, Modal identification of output-only systems using Frequency Domain Decomposition. Smart Materials and Structures, 10(3), 441-445, 2001.

[4] B. Bürki, S. Guillaume, P. Sorber, H.P. Oesch, DAEDALUS: A versatile usable digital clip-on measuring system for total stations, 2010 International Conference on Indoor Positioning and Indoor Navigation (IPIN 2010), September 15-17, 2010, Zürich, Switzerland, 2010.

[5] E. Chatzi, C. Fuggini, Structural identification of a super-tall tower by GPS and accelerometer data fusion using a multi-rate Kalman filter, Proceedings of the 3th International Symposium on Life-Cycle Civil Engineering (IALCCE 2012), October 3-6, 2012.

[6] R. Ferrari, D. Froio, E. Chatzi, C. Gentile, F. Pioldi, E. Rizzi, Experimental and numerical investigation for the structural characterization of a historic RC arch bridge, 5th ECCOMAS Thematic Conference on Computational Methods in Structural Dynamics and Earthquake Engineering (COMPDYN 2015), Crete Island, Greece, May 25-27, 2015.

[7] D. Froio, R. Zanchi, Finite element modelization and modal dynamic analyses of an historical reinforced concrete bridge with parabolic arches, M.Sc. Thesis in Building Engineering, Advisor E. Rizzi, Co-Advisor R. Ferrari, Università di Bergamo, Dipartimento di Ingegneria e Scienze Applicate, 232 pages, 2014.

[8] R. Klis, E. Chatzi, Model-based data compression for vibration monitoring using Wireless Sensor Networks, Proceedings of the 4th International Symposium on LifeCycle Civil Engineering (IALCCE 2014), Tokio, Japan, November 16-19, 2014, pp. 138145, 2014.

[9] B. Peeters, G. De Roeck, Reference-based stochastic subspace identification for outputonly modal analysis. Mechanical Systems and Signal Processing, 13(6), 855-878, 1999. 
[10] F. Pioldi, R. Ferrari, E. Rizzi, Output-only modal dynamic identification of frames by a refined FDD algorithm at seismic input and high damping. Submitted for publication, revised version, 2014.

[11] F. Pioldi, R. Ferrari, E. Rizzi, A refined FDD algorithm for Operational Modal Analysis of buildings under earthquake loading. P. Sas, D. Moens, H. Denayer Eds. 26th International Conference on Noise and Vibration Engineering (ISMA2014), Leuven, Belgium, September 15-17, 2014, pp. 3353-3368, 2014.

[12] F. Pioldi, R. Ferrari, E. Rizzi, Earthquake structural modal estimates of multi-storey frames by a refined FDD algorithm. Submitted for publication, 2014.

[13] L. Santarella, E. Miozzi, Ponti Italiani in Cemento Armato. Hoepli, Milano, 1948.

[14] A.W. Smyth, M. Wu, Multirate Kalman filtering for the data fusion of displacement and acceleration measurements. Y. Matsuzaki Ed. Proc. SPIE 6174, Smart Structures and Materials 2006: Sensors and Smart Structures Technologies for Civil, Mechanical, and Aerospace Systems, 61741G, April 11, 2006; doi:10.1117/12.658731.

[15] SVS, ARTeMIS Extractor 2011, http://www.svibs.com/, 2012.

[16] P.D. Welch, The use of Fast Fourier Transform for the estimation of Power Spectra: a method based on time averaging over short, modified periodograms. IEEE Transactions on Audio and Electroacoustics, 15(2), 70-73, 1967. 